\title{
Atrial Couplet by ECG Finding
}

National Cancer Institute

\section{Source}

National Cancer Institute. Atrial Couplet by ECG Finding. NCI Thesaurus. Code C102597.

An electrocardiographic finding in which two premature atrial complexes occur sequentially. (CDISC) 